\title{
Can Visual Design Provide Legal Transparency? The Challenges for Successful Implementation of Icons for Data Protection Arianna Rossi, Monica Palmirani
}

European Parliament and Council of European Union, Regulation (EU) 2016/679 of the European Parliament and of the Council of 27 April 2016 on the Protection of Natural Persons with Regard to the Processing of Personal Data and on the Free Movement of Such Data, and Repealing Directive 95/46/EC (General Data Protection Regulation), 0J L 119, 4.5.2016, p. 1-88, 2016. https:// eur-lex.europa.eu/eli/reg/2016/679/oj.

2 See Article 29 Data Protection Working Party, Guidelines on Transparency Under Regulation 2016/679, 17/EN WP260rev. 01 (2018). https://ec.europa. eu/newsroom/article29/document.cfm? action= display\&doc_id=51025 (accessed November 12, 2019). The Article 29 Working Party was an independent advisory body with the mission of providing expert advice to the Member States and recommendations to the European Commission about the application of data protection laws through the publication of guidelines and opinions, as well as to guarantee uniform application of the law across the EU. Under the GDPR, the work of the WP29 is continued by its replacement body, the European Data Protection Board.

3 GDPR Article 12.1.

4 For an overview of the problems related to privacy disclosures, see Arianna Rossi et al., "When Design Met Law: Design Patterns for Information Transparency," Droit de la Consommation = Consumenterecht: DCCR [Consumers protection law] 122-23 (2019): 92-7; Florian Schaub et al., "A Design Space for Effective Privacy Notices," Symposium on Usable Privacy and Security (SOUPS), Carleton University, Ottawa, Canada, July 22-24,

\section{Introduction}

In 2018, a key year for data privacy and data protection in the European Union, the General Data Protection Regulation (GDPR) became applicable. ${ }^{1}$ With it came a series of new duties and rights destined to revolutionize the ecosystem of personal data gathering and processing. The GDPR introduced a number of significant provisions that potentially produce far-reaching effects because its obligations apply to any organization offering services or goods to individuals on European soil. As a general aim, the GDPR is intended to re-establish a balance between those entities collecting and processing personal data (i.e., the data controllers) and individuals to whom that personal data belong (i.e., the data subjects), who often are unaware of the extent of the processing.

To reach this goal, the GDPR put a priority on design. The regulators assigned unprecedented relevance to the design quality of the information describing both the processing practices for personal data and the rights of the concerned data subjects. This information is commonly communicated in privacy notices. Under the GDPR, the nature, accessibility, and comprehensibility of the information describing data privacy practices must demonstrate compliance with the transparency obligations laid down in Article 12. ${ }^{2}$ The GDPR requires that any communication addressed to data subjects must be designed in a "concise, transparent, intelligible and easily accessible form, using clear and plain language." ${ }^{\prime 3}$

Such attention to design-including the modality and efficacy of data privacy communication-represents a landmark in EU data protection law. It reflects decades of research documenting the absolute incapacity of traditional privacy policies to inform people's privacy-related decisions. These traditional treatments of data privacy information take the form of lengthy, overly complex, unintelligible, and hard-to-navigate documents. ${ }^{4}$ The design of privacy communication tends to be so poor that some scholars have even categorized traditional privacy communication as dark 
2015; George Milne and Mary Culnan,

“Strategies for Reducing Online Privacy Risks: Why Consumers Read (or Don't Read) Online Privacy Notices," Journal of Interactive Marketing 18, no. 3 (2004): 15-29; and Wainer Lusoli et al., “Pan-European Survey of Practices, Attitudes and Policy Preferences as Regards Personal Identity Data Management" (Publications Office of the European Union, 2012). DOI: 10.2791/81962.

5 Dark patterns are "malicious patterns that intentionally weaken or exploit the privacy of users, often by making them disclose personal data or consent against their real interest." See Christoph Bösch et al., "Tales from the Dark Side: Privacy Dark Strategies and Privacy Dark Patterns," Proceedings on Privacy Enhancing Technologies 2016, no. 4 (2016): 242. "Good Consumer Information: The Information Paradigm at Its (Dead) End?" Journal of Consumer Policy 40, no. 2 (2017): 188.

7 WP29, Guidelines on Transparency, 5.

8 GDPR, Article 12.7

9 GDPR, Article 12.7

10 See GDPR, Article 12.8 and Article 92. See also GDPR, Recital 166, which states that "... [D]elegated acts should be adopted in respect of criteria and requirements for [...] information to be presented by standardised icons and procedures for providing such icons. It is of particular importance that the Commission carry out appropriate consultations during its preparatory work, including at expert level."

11 WP29, Guidelines on Transparency, 26 patterns-as "obscure strategies" that make it "hard or even impossible for data subjects to learn how their personal data is collected, stored, and processed." ${ }^{5}$ The GDPR challenges this dysfunction. The previous information paradigm focused on the quantity of information as a signifier of effective disclosure. ${ }^{6}$ Meanwhile, the quality of legal information design has been ignored. The Article 29 Working Party (WP29), in its guidelines on transparency maintains that the concept of transparency should be interpreted and applied in a user-centric manner. Thus, privacy notices should not just superficially comply with the legal provision on mandated disclosure, but should be effective, informative tools. Hence, the design of legal communication must account for the specificity of the intended audience and the characteristics of human cognition to provide transparent, comprehensible, and navigable disclosures.

Remarkably, the GDPR even acknowledges the potential of visual design to enhance the comprehensibility of privacy terms. Namely, it provides for the possibility of disclosing information to data subjects with text in combination with standardized visual icons to give "in an easily visible, intelligible and clearly legible manner a meaningful overview of the intended processing." ${ }^{\prime 8}$ Such icons must be machine-readable when presented in electronic format. ${ }^{9}$ Although the European Commission's role is to give directions on the creation of the icons through delegated acts, the necessity of experts' involvement is emphasized in Recital 166 of the GDPR..${ }^{10}$ In addition, the WP29, prior to any EU standardization, encourages an "evidence-based approach" and the necessity for "extensive research in conjunction with industry and the wider public as to the efficacy of icons in this context."11

The research and the open problems described in the following sections aim to contribute to the emerging debate on evidence-based design standards for data protection icons in the EU. Section 2 discusses possible explanations for the use of icons in the data protection domain by listing some advantages and disadvantages. Section 3 introduces the methodological choices for the design of DaPIS, the icon set created as a means to fulfill the GDPR's requirements. Section 4 addresses some major challenges that surfaced while designing DaPIS and advances some potential answers for further research. We focus on the object of representation of the icons, their function, the methods for their evaluation, and their interpretation.

This article also contributes to the broader discussions of design's role in effective regulation and public access to rights and laws. Can visual representations of complex technical and legal information effectively help people make sense of it-and 
12 See, e.g., Colette R. Brunschwig, "On Visual Law: Visual Legal Communication and Practices and their Scholarly Exploration," Zeichen und Zauber des Rechts: Festschrift für Friedrich Lachmayer [Signs and Magic of Law: Commemorative for Friedrich Lachmayer], ed. Erich Schweihofer et al. (Bern: Editions Weblaw, 2014): 899-933; and Volker Boehme-Nessler, Pictorial Law: Modern Law and the Power of Pictures (Berlin: Springer Science \& Business Media, 2010).

13 See Thomas Barton et al., "Successful Contracts: Integrating Design and Technology," in Legal Tech, Smart Contracts and Blockchain, ed. M. Corrales et al. (Singapore: Springer, 2019): 69-72. "Visualizations seem to have a positive impact on information finding..., understanding... and recalling...." See Stefania Passera and Helena Haapio, "Transforming Contracts from Legal Rules to User-Centered Communication Tools: A HumanInformation Interaction Challenge," Communication Design Quarterly Review 1, no. 3 (2013): 42.

14 Colette Brunschwig, "Multisensory Law and Therapeutic Jurisprudence: How Family Mediators Can Better Communicate with Their Clients," Phoenix Law Review 5, no. 5 (Summer 2012): 744.

15 On icons for the data protection domain see Samson Esayas et al., "Is a Picture Worth a Thousand Terms? Visualising Contract Terms and Data Protection Requirements for Cloud Computing Users," in International Conference on Web Engineering (Cham, Switzerland): Springer, 2016): 42; and Christopher F. Mondschein, "Some Iconoclastic Thoughts on the Effectiveness of Simplified Notices and Icons for Informing Individuals as Proposed in Article 12 (1) and (7) GDPR," European Data Protection Law Review 2 (2016): 518.

16 "Visual communication is freer and less controlled than language-based communication. ... [l]mages leave more room for internal development and interpretation.... Images are potentially more anarchic than words." BoehmeNessler, Pictorial Law, 89.

17 On hermeneutics of visual artifacts, see, e.g., Jay A. Mitchell, "Whiteboard and Black-Letter: Visual Communication take action to protect their own interests? Can design offer a means for effective participation in civic and consumer life? This article's discussion of the particular visualizations of GDPR communications provides some evidence of existing possibilities and constraints.

\section{Why Icons for the Legal Domain?}

Among the several possible visual means that can enhance the transparency of legal communication, the European legislators have overtly mentioned pictograms. But why is that? In this section, we suggest a few complementary explanations for this specific choice.

\section{Visual Design for the Legal Domain}

Visual communications of legal matters represent a rather novel, but increasingly growing field of study.12 Two opposing positions have been expressed as to how legal communications might be affected by visual design. One view highlights the attested benefits of visualizations for the communication of legal matters, mostly derived from empirical evidence. For example, visualizations of legal matters can support comprehension in the following ways: ...clarifying what written language does not manage to explain fully; making the logic and structure of the documents more visible; supporting evidence, analysis, explanation, and reasoning in complex settings; and providing an alternative access structure to the contents, especially to the non-experts working with the document. ${ }^{13}$

The other view states that, because the law is traditionally expressed through linguistic utterances (i.e., law is "verbocentric"14), visual communication poses risks. Indeed, according to this view, graphical means would not be able to transmit the nuances of legal language ${ }^{15}$ and they would be more open to interpretation than written statements. ${ }^{16}$ Thus, visual communication would augment, rather than minimize, the risks of misunderstandings. In addition, it would constitute a problem in court because no established framework or vocabulary exists for interpreting and interrogating visual legal documents, unlike the well-established legal hermeneutics for verbal provisions. ${ }^{17}$

The cautious observations of the latter view disregard three essential aspects of the actual use of visual design. First, visual elements generally are not meant to completely replace text in legal documents. ${ }^{18}$ Rather, illustrations, such as diagrams, timelines, icons, and comics, complement words..$^{19}$ Second, the aim is 
in Commercial Contracts," University of Pennsylvania Journal of Business Law 20 (2018): 837-43; and Naomi Mezey, "The Image Cannot Speak for Itself: Film, Summary Judgment, and Visual Literacy," Valparaiso University Law Review 48 (2013): 3

18 That icons, or visual elements in general, should substitute for words completely is a common misconception in the legal sphere. See, e.g., Esayas et al., "Is a Picture," 45; and Gerlinde Berger-Walliser et al., "From Visualization to Legal Design: A Collaborative and Creative Process," American Business Law Journal 54, no. 2 (2017): 349.

19 Helena Haapio and Stefania Passera, “Contracts as Interfaces: Exploring Visual Representation Patterns in Contract Design," in Legal Informatics, ed. Daniel Katz et al. (Cambridge: Cambridge University Press, 2016).

20 Haapio and Passera, Contracts as Interfaces, 14 online.

21 Connie Malamed, Visual Language for Designers: Principles for Creating Graphics That People Understand (Beverly, MA, MD: Rockport Publishers, 2009): 119

22 Sarah Isherwood et al., "Icon Identification in Context: The Changing Role of Icon Characteristics with User Experience," Human Factors 49, no. 3 (2007): 465. For a critical examination of the supposed universality of icons, see, e.g., Robert Dewar, "Design and Evaluation of Public Information Symbols," in Visual Information for Everyday Use: Design and Research Perspectives, ed. Harms Zwaga et al. (London: Taylor \& Francis, 1999), 285-303.

23 Isherwood et al., "Icon Identification in Context," 467.

24 Margaret Hagan, "Rethinking Data Privacy Communication Design: Three Big Questions from Bologna," Legal Design and Innovation (website) (2018), https://medium.com/legal-designand-innovation/rethinking-data-privacycommunication-design-3-big-questionsfrom-bologna-13275a987047 (accessed November 11, 2019).

25 https://creativecommons.org/shareyour-work/licensing-considerations/ (accessed February 10, 2020).

26 For traffic signs, warning signs, and labeling schemes for energy not to have visual elements represent legal meanings as precisely as verbal expressions can do. Instead, they can clarify, give salience to, and improve memorability and navigability of information-for instance, by making visible abstract relations between concepts (e.g., sequences or conditions) that are typical of legal documents. ${ }^{20}$ In the legal domain, then, clarifying that different kinds of visual elements convey different types of information and adopt different functions is important; some of these functions are not inherently pictorial. For example, timelines illustrate temporal sequences and comic strips can properly represent narratives, while companion icons can support strategic reading in long documents.

Users' interpretation of legal documents does not correspond to the hermeneutical activity of legal professionals. Whereas the latter is a specific methodology for the interpretation of legal provisions, the former is a regular communicative process finalized to the understanding of a (linguistic or non-linguistic) message. Although we recognize the need for extensive research on the first aspect, we focus in this article on the latter.

\section{Icons for the Legal Domain}

As simplified visual illustrations, icons cannot enhance comprehensibility of data practices as other visual elements that involve complex content (e.g., videos or comics) could do. However, they can be recognized, processed, and memorized with ease and thus can serve as cognitive support for the classification of content better than text can, as graphic user interfaces successfully demonstrate. ${ }^{21}$ In addition, we note a widespread belief that icons can overcome linguistic and cultural barriers, which also is commonly held in the juridical domain. ${ }^{22}$ Whereas this belief holds true for standardized conventions (e.g., the traffic signs and the graphical symbols used in public spaces) and for icons representing concrete objects, the meaning of symbols that are not semantically transparent must be learned rather than deduced. ${ }^{23}$

However, given the verbo-centricity of the law, icons are less disruptive non-linguistic elements than comics and other possible visual mechanisms that would completely transform legal notices. ${ }^{24}$ Moreover, well-accepted examples of pictograms used as universal shorthand for critical legal-technical information do exist. These examples include the pictograms of Creative Commons licenses for intellectual property. ${ }^{25}$ Other widely used and even internationally standardized symbols include traffic signs, warning signs, and labeling schemes for energy consumption. ${ }^{26}$ Other popular pictograms symbolize notions related to cybersecurity (e.g., the padlock for secure communications and connections) 
consumption, respectively, see United Nations Economic Commission for Europe (UNECE), https://www.unece.org/fileadmin/DAM/trans/conventn/Conv_road_ signs_2006v_EN.pdf (accessed February 10, 2020); UNECE, “Globally Harmonized System of Classification and Labelling of Chemicals," GHS Rev. May 8, 2019, https://www.unece.org/index. php?id= 51896\&L=0 (accessed February 10, 2020); and European Commission (website), About the Energy Label and Ecodesign, https://ec.europa.eu/ info/ energy-climate-change-environment/ standards-tools-and-labelsproducts-labelling-rules-and-requirements/ energy-label-and-ecodesign/about_en (accessed February 10, 2020).

27 For a discussion on the standardization of data protection icons, see Arianna Rossi and Gabriele Lenzini, "Making the Case for Evidence-Based Standardization of Data Privacy and Data Protection Visual Indicators," JOAL, Special Issue on "Visual Law," Rossana Ducato, guest ed., Vol. 8, no. 1 (2020), ISSN: 2372-7152. Open access at: https://ojs.law.cornell. edu/index.php/joal/article/view/103.

28 See, e.g., Mary Rundle, "International Personal Data Protection and Digital Identity Management Tools," Berkman Center Research Publication No. 200606), https://papers.ssrn.com/sol3/papers. cfm?abstract_id=911607; Matthias Mehldau, Iconset, “Data-Privacy Declarations v0.1," https://netzpolitik.org/wpupload/data-privacy-icons-v01.pdf laccessed February 10, 2020; Joshua Gomez et al., KnowPrivacy (website), "Privacy Coding Methodology" (2009), http://knowprivacy.org/policies_methodology.html; Renato lannella and Adam Finden, "Privacy Awareness: Icons and Expression for Social Networks," 8th International Workshop for Technical, Economic and Legal Aspects of Business Models for Virtual Goods, incorporating the 6th International Open Digital Rights Language Workshop, September 31October 1, 2009, Namur, Belgium, eds. Alapan Arnab and Jean-Noël Colin (Namur: Presses universitaires de Namur, 2010), 1-15; Privacy Icons (web page) https://wiki.mozilla.org/Privacy_Icons (updated June 28, 2011; " Final HCl Research Report," ed. Cornelia Graf et al., Primelife Deliverable D4.1.5 (2011), and to data access permissions (e.g., the geolocation symbol). In all these cases, the rationale supports the creation of a common pictographic system that can become universally recognizable when used consistently. ${ }^{27}$

A few initiatives for the creation of an icon language to summarize data practices exist, although they have neither gained acceptance nor reached extensive adoption. ${ }^{28}$ Two European-led efforts are of note. The first was conducted as part of the European PrimeLife project, ${ }^{29}$ which is the most structured attempt to create and assess icons for the data protection domain in the EU. The second presented six icons and their description in table format and was included in the 2013 Draft report on the GDPR proposal..$^{30}$ The display of such icons would have constituted a legal obligation for data controllers if the amendments had been approved. Although the icons were ultimately discarded, traces of this proposal can be found in the GDPR's call for icons.

\section{Methodology for the Design of DaPIS}

Following the GDPR's revamped interest for pictograms as transparency-enhancing means and taking stock of the lessons derived from the few previous attempts to design privacy icons, our research group drafted the DaPIS (Data Protection Icon Set), an icon set representing core concepts of EU data protection law. ${ }^{31}$

\section{An Ontological Foundation}

In the creation of DaPIS, we followed participatory design methods and structured it toward the goal of integration with semantic technologies. DaPIS was modeled on a specific, formal conceptualization of EU data protection law; $; 2$ and it represents key notions grouped in categories, such as the rights of the data subjects and the purposes of data processing. The meaningful combination of these legally significant categories can support a uniform visual design scheme.

Our team deliberately created the icon set to be modular, systematic, and semantic, so that it was not just a visual design intervention, but an intelligent one. The visual signs representing fundamental concepts (e.g., right, withdraw, consent) can be combined to express complex legal meanings (e.g., the right to withdraw consent) in the same pictogram. We primarily used the root/ referent icon design approach, where the root is a constant symbol representing the category, while the referent specifies the subcategory. ${ }^{33}$ We thereby ensured visual uniformity among the icons belonging to the same class, to ease their recognition. For instance, an upward-facing hand distinguishes the icons depicting the rights of the data subjects from the other conceptual classes (see Figure 1). 
Figure 1

DaPIS pictograms representing the various rights of the data subject and showing the modularity of the icon set: a) rights of the data subject; b) right to be informed;

c) right to rectification; d) right to erasure; e) right of access; f) right to data portability; g) right to object to processing; h) right to restriction to processing; i) right to withdraw consent; j) right to lodge a complaint to the supervisory authority. Figure created by the authors. The icons have been released under a Creative Commons Attributions-ShareAlike 4.0 International License.

http://primelife.ercim.eu/images/stories/ deliverables/d4.1.5-final_hci_research_ report-public.pdf; European Parliament, “Compromise amendments on Articles 1-29." COMP Article 1. 07.10.2013 (2013): 30-32, https://www.europarl. europa.eu/meetdocs/2009_2014/documents/libe/dv/comp_am_art_01-29/ comp_am_art_01-29en.pdf (accessed February 12, 2020); TrustArc (blog),

"TRUSTe and Disconnect Introduce Visual Icons to Help Consumers Understand Privacy Policies" (2014), https://www. trustarc.com/blog/2014/06/23/truste-disconnect-introduce-visual-icons-to-helpconsumers-understand-privacy-policies/ (accessed November 11, 2019). (The icons are no longer available.); PrivacyTech (website), "Privacy Icons" (2017) https://www.privacytech.fr/privacyicons/ (accessed February 12, 2020); and Louisa Specht-Riemenschneider and Linda Bienemann, "Informationsvermittlung durch standardisierte Bildsymbole ein Weg aus dem Privacy Paradox?" [Communication of information through standardized symbols - a way out of the privacy paradox?] in Datenrecht in der Digitalisierung [Data Law in Digitalisation], ed. Louisa Specht-Riemenschneider et al. (Berlin: Erich Schmidt Verlag, 2019).

29 See, e.g., Graf, Final $\mathrm{HCl}$ Research Report.

30 See, e.g., European Parliament, Compromise amendments.

31 For further details about the design of DaPIS, see Arianna Rossi and Monica Palmirani, "What's in an Icon? Promises and Pitfalls of Data Protection Iconography," in Data Protection and Privacy: Data Protection and Democracy, ed. Dara Hallinan et al. (Oxford: Hart Publishing:

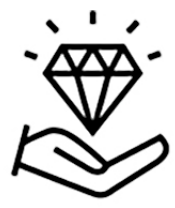

a)

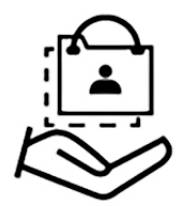

f)

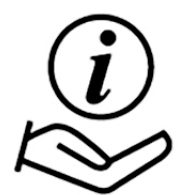

b)

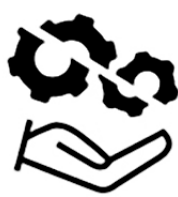

g)

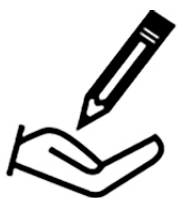

c)

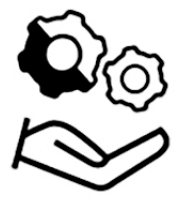

h)

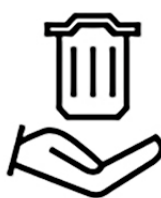

d)

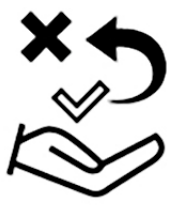

i)

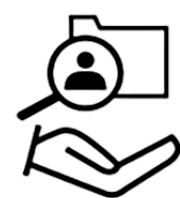

e)

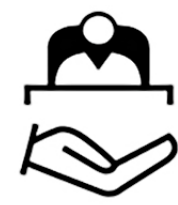

j)

The ontological foundation was also instrumental for the creation of a machine-readable icon set (as enshrined by GDPR Article 12.8) - that is, an icon language whose elements have computerinterpretable meanings that are explicitly and formally defined in the ontology. This capability allows for semi-automatic retrieval and display of the visualizations encoded in the ontology after the semantic expressions of the privacy policy in natural language (e.g., "you," "user") have been associated with their corresponding ontological class (e.g., "data subject") through an Extensible Markup Language (XML) mark-up. ${ }^{34}$ The mark-up elements also allow for a structured, semantically enriched document layout that improves its information architecture: It allows for visualizing structural elements that convey information hierarchy and thereby facilitate the reading (or, more accurately, skimming) activity. Our vision was that semantically enriched privacy policies can be leveraged to generate a user-friendly visual layer composed of structured layout and icons that can ease the navigation of these documents and increase comparability across them, both for human beings and intelligent systems.

\section{Semiotic Considerations}

To create DaPIS, a communicative and semiotic consideration of design was adopted because "one of the principal functions of design is to communicate. ${ }^{\prime 35}$ Design in this perspective is a dialogue between designer and intended user. Hence, it is not a monodirectional but a bidirectional process. Given "the existence of expressive intent and interpretative response," design is a form of mediated, asynchronous communication. ${ }^{36}$ Like written communication, the interpretation of the message embedded in the artifact (e.g., icon, button, visualization) is carried out in a different time and place than its production. The designer tries to encode a specific meaning in an artifact (like an icon) so that final users can 


\section{0); Arianna Rossi and Monica}

Palmirani, "DaPIS: An Ontology-Based

Data Protection Icon Set," in Knowledge

of the Law in the Big Data Age: Frontiers

in Artificial Intelligence and Applications,

eds. Ginevra Peruginelli and Sebastiano

Faro, Volume 317 (Amsterdam: IOS

Press, 2019), 181-95, DOI: 10.3233/

FAIA190020; Arianna Rossi, "Legal

Design for the General Data Protection

Regulation: A Methodology for the

Visualization and Communication of

Legal Concepts" (PhD thesis, Alma Mater

Studiorum Università di Bologna; $\mathrm{PhD}$

in Law, Science and Technology 2019):

Chapter 6.

32 For a thorough description of the data protection ontology PrOnto and its goals, see Monica Palmirani et al., "PrOnto: Privacy Ontology for Legal Reasoning," in International Conference on Electronic Government and the Information Systems Perspective, eds. Andreas Kö and Enrico Francesconi, Lecture Notes in Computer Science, vol. 11032 (Cham, Germany: Springer, 2018): 139-52. DOI: 10.1007/ 978-3-319-98349-3_11.

33 Lisa Fontaine et al., "Signs That Work, Phase 2: Symbol Design Research Report," (2010): 8; http://www.healthdesignnetwork.net/s/2-UHCS-ResearchReport.pdf (accessed February 12, 2020).

34 Akoma Ntoso (http://www.akomantoso. org) is a legal open XML standard for legislative, judiciary, and legal documents. See Monica Palmirani and Fabio Vitali, "Akoma-Ntoso for Legal Documents," in Legislative XML for the Semantic Web, ed. Giovanni Sartor et al. (Dordrecht: Springer, 2011): 75-100.

35 Woodrow Hartzog, Privacy's Blueprint: The Battle to Control the Design of New Technologies (Cambridge, MA: Harvard University Press, 2018): 27. For a general review of communicative and semiotic considerations of design, see Clarisse Sieckenius de Souza, The Semiotic Engineering of Human-Computer Interaction (Cambridge, MA: MIT Press, 2005).

36 Nathan Crilly et al., “Design as Communication: Exploring the Validity and Utility of Relating Intention to Interpretation," Design Studies 29, no. 5 (2008): 425-27.

37 Crilly et al., "Design as Communication," 442.

38 Mondschein, "Iconoclastic Thoughts," 515. correctly decode the intended meaning (e.g., the icon's function) through their interaction with the artifact. However, users do not have direct access to the original intentions of the designer, who must therefore be able to anticipate any problematic interpretation that would lead to misunderstandings, frustration, or errors. Ultimately, the interpretation, rather than the intention, is what determines success of use of a certain design..$^{37}$

This asynchronous interpretation matters greatly for legal design. In the design of information, graphics, interfaces, and systems, the problem of mediated communication acquires even deeper significance if the actions taken by a user based on her understanding of the artifact have legal consequences. Incorrect interpretation of interface elements, including icons, toggle bars, and buttons, might cause users to unintentionally give consent to privacy-invasive practices. Indeed, some legal scholars have voiced fears of misjudgments: Mondschein has maintained that boiling down complex legal disclosures to a set of icons would affect their quality and explanatory nature, more than correcting for information overload..$^{38}$ Misrepresentations also constitute a risk, when the visual translation of complicated processes is limited by predefined and potentially inappropriate categories or elements. The few existing user studies carried out on the interpretation of privacy icons have demonstrated that sign reception can be misguided. ${ }^{39}$

Therefore, as a crucial cautionary element, our team has prioritized an "evidence-based approach," with the aim of providing a rigorous assessment of the efficacy of icons as legal transparency mechanisms ${ }^{40}$ Because images, and especially pictograms, are polysemic, establishing whether they convey the intended message to the audience is necessary. Icon interpretation is a non-linear task and depends both on context and on the extent to which the repertoire of signs of designers and users correspond. ${ }^{41}$ To align designers' intentions and users' interpretation, we have relied on participatory design methods in the phases of conception and creation of the icons.

\section{Participatory Design Methods}

EU regulators have not provided any indication about the modality of implementation of the GDPR's icons; meanwhile, the European Commission has deliberately let solutions arise in a bottom-up manner, from civil society and industry, before adopting a binding act that imposes EU standardization. ${ }^{42}$ However, this approach has caused a lack of uniformity among the existing approaches, which results in weak incentives for the adoption of and investment in 
39 See, e.g., Leif-Erik Holtz et al., "Towards Displaying Privacy Information with Icons," in IFIP PrimeLife International Summer School on Privacy and Identity Management for Life, ed. Simone Fischer-Hübner et el. (Berlin, Heidelberg): Springer, 2010): 338-48; John Sören Pettersson, "A Brief Evaluation of Icons in the First Reading of the European Parliament on COM (2012) 0011," in IFIP International Summer School on Privacy and Identity Management (Springer, 2014): 125-35; and lannella et al., "Privacy Awareness," 1-15.

40 WP29, Guidelines on Transparency, 26.

41 Ryan Abdullah and Roger Hübner, Pictograms, Icons \& Signs: A Guide to Information Graphics (New York: WW Norton, 2006), 14.

42 Directorate General Justice and Consumers, European Commission, private communication reported in Serge Tagne, Transparence dans le RGPD. Les icônes tiendront-elles la promesse? [Transparency in the GDPR. Will the icons keep the promise?], thesis, ISEP (2018): annex 1.

43 Joel Reidenberg et al., "Trustworthy Privacy Indicators: Grades, Labels, Certifications, and Dashboards," Washington University Law Review 96 (2019): 1409.

44 See, e.g., Maja van der Velden and Christina Moertberg, "Participatory Design and Design for Values," in Maja van den Hoven et al., Handbook of Ethics, Values and Technological Design (Dordrecht: Springer, 2015): 41-66. See also Arianna Rossi and Helena Haapio, "Proactive Legal Design: Embedding Values in the Design of Legal Artefacts," in Internet of Things: Proceedings of the 22nd International Legal Informatics Symposium IRIS 2019, ed. Eric Schweighofer et al. (Vienna: Editions Weblaw, 2019): 537-44.

45 The first workshop was held in July 2017 at the Legal Design Lab of Stanford Law School, Stanford, CA. Subsequent workshops were organized over the course of 2018 at the CIRSFID (Interdepartmental Centre for Research in the History, Philosophy, and Sociology of Law and in Computer Science and Law) of the University of Bologna (Italy) in collaboration with the Academy of Fine Arts of Bologna and the Associazione Italiana Informatica Giuridica.

46 For instance, for computer scientists the prototypical representation of data is a privacy indicators, and in a proliferation of differing icon sets. This inconsistent visual design hinders users' abilities to easily recognize icons and rely on them for guidance on the law and their rights. ${ }^{43}$

We designed DaPIS using participatory design methods with two purposes in mind: to allow for the expression of multifaceted values and priorities of the different stakeholders who might be affected by the icon set and to avoid overlooking any fundamental aspect of legal icon design ${ }^{44}$ We held a series of workshops involving various stakeholders (i.e., a heterogeneous group of graphic designers, lawyers and legal scholars, computer scientists, communications professionals, interested laypeople, and representatives of the business world), with the intention of combining their different visions. ${ }^{45}$ The preparatory, conceptual work for the design of the graphical symbols involved mind-mapping techniques to gather a wide choice of motifs for each preselected legal notion.

For instance, graphic professionals proposed the root/referent icon design approach and sought to ensure the quality and overall coherence of the visual design. They provided plausible contexts of use for the icons. Meanwhile, legal experts and computer scientists guided the interpretation of the abstract legal-technical definitions described in the GDPR. Moreover, individuals from for-profit business enterprises offered a critical voice on the expected hurdles to the implementation of the icons in the market. Laypeople offered a non-specialized view that supported the development of universally understandable symbols, as opposed to graphical conventions known only to professionals. ${ }^{46}$

Involving multiple stakeholders also underlined crucial differences among their views and priorities. One of the most evident divergences concerned expectations about the visual representations of legal notions: Whereas legal scholars defended the importance of a literal and detailed "visual translation" of the concepts to avoid their misrepresentation and oversimplification, designers emphasized the crucial relevance of criteria like simplicity and legibility of the icons to support ease of recognition and the ability to render them on a variety of devices and screen sizes. Collaborative prototyping enabled the different stakeholders to negotiate their views in a shared design space and to reach a satisfactory mediation. ${ }^{47}$ The final DaPIS comprises 37 elements. ${ }^{48}$

\section{Open Questions and Problems}

During the development of the research, a series of open questions emerged, and we propose these questions as a guide for future work in visual design for legal transparency. 
cylinder, while for laypeople, the file folder is a more recognizable symbol. See Arianna Rossi and Monica Palmirani, "From Words to Images Through Legal Visualizations," in Al Approaches to the Complexity of Legal Systems, ed. Ugo Pagallo et al. (Cham: Springer): 80.

47 Van der Velden and Moertberg, Participatory Design, 59.

48 DaPIS is available for download at http://gdprbydesign.cirsfid.unibo.it/ dapis-2/ (accessed November 11, 2019) and is licensed under a Creative Commons Attributions-ShareAlike 4.0 International License.

49 As in the cases of Mehldau, Iconset; lannella et al., Privacy Awareness; Holtz et al., Towards Displaying; PrivacyTech, Privacy Icons; SpechtRiemenschneider and Bienemann, Bildsymbole [Pictograms].

50 As in the case of Moskowitz and Raskin, Privacy Icons.

51 As in the cases of Rundle, "International Personal Data Protection"; European Parliament, "Compromise Amendments (LIBE) Committee, Draft Report.

52 Zohar Efroni et al., "Privacy Icons: A Risk-Based Approach to Visualisation of Data Processing," European Data Protection Law Review 5, no. 3 (2019): 352-66; and Max von Grafenstein et al., “Designing Privacy Icons \& Testing for its Effectiveness by an Interdisciplinary Research Methodology" (2019), https:// privacyiconsforum.eu/projects/designingprivacy-icons-and-testing-for-its-effectiveness/ (accessed January 22, 2020).

53 The issue of the icons' object of representation has been more extensively explored in Rossi and Palmirani, "What's in an Icon?": 69-70.

54 See, e.g., Susan Wiedenbeck, "The Use of Icons and Labels in an End User Application Program: An Empirical Study of Learning and Retention," Behaviour \& Information Technology 18, no. 2 (1999): 68-82.

55 For a collection of transparency-enhancing design patterns (including but not limited to icons) for privacy notices, see Rossi et al., "When Design Met Law," 99-120.

\section{The Challenge of the Object of Representation}

One fundamental question concerns the objects that the visual language should represent. Previous design efforts fall into three approaches to object representation. The first focuses on single objects and concepts that are proper to the privacy and data protection domain (e.g., the concept of "pseudonymization" or that of "encryption"). ${ }^{49}$ The second tries to visually represent statements about such concepts, referring to the presence of a certain data practice (e.g., "Site contains third-party ads" ${ }^{\prime 50}$ ). The third approach includes attempts to give an indication of the lawfulness of specific data practices (e.g., "No personal data are collected beyond the minimum necessary for each specific purpose of the processing" $\left.{ }^{\prime \prime 1}\right)$. The aim here is to rate such practices to provide meaningful advice and to inform users' decisions on whether to use a certain service-or to head elsewhere. Similarly, other approaches put an emphasis on risky data processing aspects..$^{52}$

This problem and question introduces an additional critique about the icons' fit to represent knowledge in law. ${ }^{53}$ Icons are generally best fit to depict concrete concepts, such as objects and people. Abstract data protection notions (e.g., "processing purposes") are inherently difficult to visualize and to decode. Individuals must resort to contextual elements, previous experience, and learned knowledge to correctly interpret them. For this reason, supplementing icons with textual labels or other interface design elements can explain their meaning and therefore facilitate their interpretation. ${ }^{54}$ Such elements are necessary at first exposures in cases where the relationship between the graphical symbol and its meaning is arbitrary and cannot be inferred. Therefore, expectations of what icons can do, when based on the ways icons have been used to symbolize concrete concepts, are inappropriate in this case, and the expectations are what must be reviewed. It is only by providing enough interpretative context, preferring concrete concepts over abstract ones and actively supporting the learning of the association between pictogram and meaning that icons can aspire to communicate universally and univocally. However, icons represent only one of the possible solutions to the endemic lack of transparency in privacy notices..$^{55}$

Another critique of the use of icons to clarify legal concepts moves from the fact that these graphical symbols are not suitable to communicate the nuanced notions expressed in legal terms. The legal experts that took part in DaPIS's participatory workshops expected to accurately translate the legal definitions into their visual equivalents by preserving the sheer amount of details and the complexity that characterize legal provisions. The 
56 Jaspreet Bhatia et al., "A Theory of Vagueness and Privacy Risk Perception" (24th International Requirements Engineering Conference (RE), Beijing, 2016), IEEE Xplore, 26, DOI: 10.1109/ RE.2016.20.

57 "'Third party' refers to a natural or legal person, public authority, agency or body other than the data subject, controller, processor and persons who, under the direct authority of the controller or processor, are authorized to process personal data," GDPR Article 4.10.

58 The topic of the icons' function has been more thoroughly discussed in Rossi and Palmirani, "What's in an Icon?":

72-75 and in Arianna Rossi and Gabriele Lenzini, "Which Properties has an Icon? A Critical Discussion on Evaluation Methods for Standardised Data Protection Iconography," in Proceedings of STAST (Berlin, Heidelberg: Springer, forthcoming), Section 5 .

59 See Abdullah and Hübner, Pictograms, $17,30$.

60 See the companion icon pattern in Haapio and Passera, "Contracts as Interfaces," 26; see also related examples in Rossi et al., "When Design Met Law," 105, 108-109.

61 Behavioural Insights Team, Best Practice Guide: Improving Consumer Understanding of Contractual Terms and Privacy Policies: Evidence-based Actions for Businesses (London: Behavioural Insights, 2019), 11-12, https://assets. publishing.service.gov.uk/government/ uploads/system/uploads/attachment_ data/file/831400/improving-consumerunderstanding-contractual-termsprivacy-policies.pdf. The work was commissioned by the Department of Business, Energy and Industrial Strategy of the United Kingdom. underlying hypothesis predicted that the addition of more traits and symbols to a pictogram would improve icon comprehension. In addition, the jurists firmly supported a literal translation of the concepts into the pictograms to decrease the set of plausible interpretations to one univocal meaning. Informed by this position, the initial prototypes of DaPIS resulted in complex and detailed pictograms. However, our user studies revealed that literal, precise representations appeared confusing and overwhelming to the users, instead of representing meaningful guidance.

Remarkably, even the opposite problem was encountered: Some concepts lack a precise definition, not only because natural language is ambiguous in itself, but also because legal and, in particular, privacy terms are deliberately left vague to be open to interpretation. ${ }^{56}$ For instance, data processing can be necessary to provide a certain service (e.g., a maps app needs the user's geolocation data to guide her to the desired destination). Thus, legal expressions, such as "we use the data we collect to provide you with the information and services that you requested from us," constantly figure among the processing purposes of a service provider but is not further specified. Visualizing such a vague "purpose of provision of the service" has thus represented a challenge. An emblematic and extreme case also is represented by the concept of "third party," which is a fundamental concept in data protection regulation and is legally defined by what it is not, instead of by what it is. ${ }^{57}$ For these reasons, similar abstract and loose legal notions were difficult to translate into easily interpretable visuals.

\section{The Challenge of Defining Icon Functions ${ }^{58}$}

The diversity concerning the icons' object of representation, as described, also is reflected in the different functions that an icon set can assume related to transparency in privacy disclosures..$^{59}$ Graphical symbols depicting individual notions can accompany headings or key points of the notice to saliently indicate where specific information can be found. These "companion icons" are meant to break the wall of text and thereby to attract readers' attention and help them to skim through the document to efficiently identify specific information. ${ }^{60}$ Evidence shows that they can increase readers' comprehension of privacy policies. ${ }^{61}$ This design pattern can be particularly advantageous in lengthy documents that are devoid of an information hierarchy.

Symbols that try to unequivocally communicate to users what privacy practices are stated or are absent from a privacy policy add a layer of meaning to companion icons. For instance, visual 
62 "The data subject shall have the right not to be subject to a decision based solely on automated processing, including profiling, which produces legal effects concerning him or her or similarly significantly affects him or her," GDPR Article 22.

63 "Personal data shall be: ....adequate, relevant and limited to what is necessary in relation to the purposes for which they are processed (data minimisation)," GDPR Article 5(1)(c).

64 See Privacy Icons, "Disconnect." See Specht-Riemenschneider and Bienemann, "Informationsvermittlung."

65 See Rossi and Palmirani, "What's in an Icon?": 72-73.

66 Pedro Giovanni Leon et al., "Privacy and Behavioural Advertising: Towards Meeting Users' Preferences," (Symposium on Usable Privacy and Security (SOUPS), Carleton University, Ottawa, Canada, July 22-24, 2015) symbols can signal that profiling of the data subject occurs or that personal data are anonymized. Whether this practice respects the user's privacy preferences or not is left to the user to discern.

A system of icons also can attract users' attention to specific data practices that can be considered risky (e.g., automated decision-making that has significant legal implications for the data subject ${ }^{62}$ ) or unlawful (e.g., processing a larger amount of data than necessary, thus contradicting the principle of data minimization ${ }^{63}$ ). In this view, icons assume the role of warning signs, like those indicating explosive or poisonous materials, those signaling the security or insecurity of an internet connection, or those communicating a potential risk to the driver. Conversely, visual elements that act as "quality seals" and highlight good practices (e.g., "Processing of data within Europe or a third country with a sufficient level of data protection" ${ }^{\prime 64}$ ) also can be very informative for users' decisions about their privacy.

Given the ontology of concepts used as a methodological framework to create the icons, DaPIS depicts individual concepts that cover the different ontological classes. This choice allows practitioners and researchers to devise and explore automated or semi-automated concept-mining techniques that recognize where a certain subject is described in a text and that display the corresponding icons, serving the function of information-markers. However, this approach is feasible only in standardized, wellstructured privacy policies, where each thematic section covers one topic. Moreover, the adoption of companion elements reflects a deliberate, cautious position about the interpretability of icons. Instead of trying to completely replace the legal text, the aim is to attract the data subject's attention and to aid in the navigation of long legal documents, thus supporting the reader's interpretation through a combination of textual and pictorial cues.

Another fundamental reason to adopt icons representing individual concepts is that providing any kind of decontextualized judgment about the lawfulness or riskiness of a legal practice might be problematic. ${ }^{65}$ Sentence-level icons arguably could be more informative and thus more helpful for data subjects' privacyrelated decisions, but they also would entail an interpretation about the goodness of such practices and thus would interfere with the autonomy and self-determination of individuals. Moreover, indicating the riskiness of a certain practice per se and a priori can be a questionable choice, given that context is key to determine the level of risk. For example, profiling might be problematic if used for price discrimination, but it might be considered useful and even desirable if aimed at providing targeted special offers. Moreover, research has demonstrated that privacy preferences vary greatly ${ }^{66}$; what is considered invasive by one person might be considered acceptable by another. 
67 Reidenberg et al., "Trustworthy Privacy Indicators," 16.

68 See TOS;DR, Classification, https://tosdr. org/classification.html (accessed January 24, 2019).

69 Hamza Harkous et al., "Polisis: Automated Analysis and Presentation of Privacy Policies Using Deep Learning," arXiv:1802.02561v2 (2018).

70 See, e.g., Efroni et al., "Privacy Icons: A Risk-Based Approach"; and von Grafenstein et al., "Designing Privacy Icons."

71 For a discussion on icon evaluation methods and measures, see Rossi and Lenzini, "Which Properties has an Icon?" See also Siné J.P. McDougall et al., "Measuring Symbol and Icon Characteristics: Norms for Concreteness, Complexity, Meaningfulness, Familiarity, and Semantic Distance for 239 Symbols," Behavior Research Methods, Instruments, \& Computers 31, no. 3 (1999): 487-519.

72 See Malamed, Visual Language for Designers, 118; and Jon Hicks, The Icon Handbook (Cardiff: Five Simple Steps, 2011), 22.

73 Isherwood, Icon Identification in Context, 467.

74 See, e.g., European Telecommunications Standards Institute, Human Factors (HF); Framework for the Development, Evaluation and Selection of Graphical Symbols. EG 201379 V1.1.1 (1998-12); and ISO, ISO 9186-1:2014. Graphical symbols-Test methods-Part 1: Method for testing comprehensibility, https:// www.iso.org/standard/59226.html (accessed February 12, 2020).
In addition, even the adoption of such icons by data controllers might be troublesome. The GDPR states that the data controller decides whether to use icons in combination with written information to comply with the transparency obligation. Expecting that a service provider would deliberately warn its users about practices that they would find unfavorable is unreasonable. ${ }^{67}$ However, third-party services that provide visual indicators for the data protection practices of data controllers offer an alternative solution. For instance, Terms of Service; Didn't Read (ToS;DR) uses crowdsourcing to analyze privacy policies and so to provide the visual ratings ${ }^{68}$; meanwhile, Polisis uses deep learning. ${ }^{69}$ Both third-party solutions can be contested because they reflect mediated interpretations (by non-expert humans and by artificial intelligence that was trained on manually annotated data, respectively) and might therefore be subject to error. However, some scholars maintain that this approach represents a viable manner to implement an actual "informed consent" and are starting to investigate this research direction. ${ }^{70}$

For all these reasons, a multi-stakeholder discussion with policy-makers, the public, and regulated organizations is advisable. The European Commission, service providers, citizens, consumer associations, practitioners, and researchers and scholars from disciplines including design, philosophy of law, psychology, behavioral economics, and neuroscience should be involved in determining the function that GDPR icons should have, according to the function they intend to serve and goal they intend to achieve.

\section{The Challenge of Icon Evaluation ${ }^{71}$}

Icons do not necessarily foster comprehension of the concepts they represent, although many assume they do. Ease of an icon's interpretation depends on well-defined characteristics, such as semantic distance (also defined as level of arbitrariness). Concrete icons are easily recognizable even at users' first exposures; meanwhile, the meaning of arbitrary icons has to be learned rather than inferred..$^{72}$ In the latter case, immediate comprehension is impossible to reach: Rather, as familiarity increases with repeated exposures, recognition rates do as well. In addition, familiarity has a dual nature ${ }^{73}$ : It involves both previous knowledge of the concept (e.g., the concept of "geolocalization") and previous experience with its visual representation (e.g., the omnipresent pin icon). Furthermore, because individual characteristics, such as cultural background, age, and domain expertise, affect how knowledgeable users are in the legal and technical area, they also can influence ease of icon interpretation.

Such factors challenge standard international methods of icon evaluation, which are appropriate only if the concept represented in the icon is known to the interpreters. ${ }^{74}$ The ISO standard 
75 See Rossi, Legal Design for the General Data Protection Regulation, 271-73; and Rossi and Lenzini, "Which Properties Has an Icon," 15. See also ISO, ISO 9186-3:2014. Graphical symbols - Test methods Part 3: Method for testing symbol referent association, https:// www.iso.org/standard/59882.html (accessed February 12, 2020).

76 On the issue of usability evaluation methods, see, e.g., Saul Greenberg and Bill Buxton, "Usability Evaluation Considered Harmful (Some of the Time)," $\mathrm{CHI}$ '08: Proceedings of the SIGCHI Conference on Human Factors in Computing Systems (New York: ACM, 2008), 111-120, https://doi. org/10.1145/1357054.1357074.

77 See Jennifer Snow Wolff and Michael S. Wogalter, "Comprehension of Pictorial Symbols: Effects of Context and Test Method," Human Factors 40, no. 2 (1998): 173-86.

78 For a critical examination of user studies about the comprehensibility of privacy icons, see, e.g., Rossi and Lenzini, "Which Properties Has an Icon," 4. for testing symbols whose referents are unknown also presents some limitations, as we have maintained elsewhere. ${ }^{75}$ Such evaluation does not measure the learnability of an icon system in context and is exclusively based on quantitative methodologies. Longitudinal studies using a mixed methods approach would probably be more informative about the effectiveness of icons and more methodologically sound. ${ }^{76}$

Providing contextual cues that mirror the actual use situation of the icons is crucial to ease the interpretation process during icon assessment by users. Without taking into consideration familiarity and without providing the intended context of use, low recognition scores would mistakenly indicate that re-design and further testing are necessary. ${ }^{77}$ Indeed, the few existing studies on the efficacy of data protection icons have overlooked such dimensions; as a result, most of the visual elements have been discarded, based on the low recognition rates of icons that represent unfamiliar concepts or that are displayed without sufficient context. ${ }^{78}$

Appropriate evaluation techniques should be used to determine whether icons are effective in other roles in legal contexts. If icons are to be used as navigation cues in privacy policies, then the need is to evaluate whether users can find specific pieces of information in these documents (i.e., effectiveness); whether they can do so more easily, or more quickly (i.e., efficiency); and whether they give a better user experience (i.e., more satisfaction and less frustration) than in text-only documents. If icons should unambiguously indicate the presence or absence of a certain data practice, then there should be evaluation as to whether users comprehend these dualities. If icons should warn users against risky or unfair data processing, the evaluation focuses on their noticeability and their influence on users' decision-making process (e.g., the choice of a certain service over another).

Our team has evaluated the DaPIS icons' legibility and comprehensibility. Legibility assessment concerns the ease of recognition of the single elements that compose the icons and influences the ease of recognition of the icon as a whole. We established two evaluation criteria for this comprehensibility assessment: first, a subjective estimation of the fit for correspondence between visual representation and underlying concept; and second, whether the interpreter was able to speculate about the underlying motivations for a certain icon choice, even if its meaning was not immediately comprehensible at the first exposure.

The overall results indicate that the icons with higher levels of concreteness and familiarity are more easily recognizable, while those that try to represent abstract or unfamiliar notions were difficult to understand. The results provide a first, elementary indication of which visual elements are more recognizable and which 
79 Three research studies were carried out on subsequent, revised versions of DaPIS, but with a small pool of mostly young and well-educated users. For a detailed illustration, see Rossi and Palmirani, "What's in an Icon?": 77-80; and Rossi, Legal Design for the General Data Protection Regulation, Chapter 6.

80 Rossi and Lenzini, "Which Properties Has an Icon," 11-3.

81 GDPR Article 12.7.

82 In the WP29 guidelines, for example, they should be "universally used and recognized across the EU as shorthand for information." WP29, Guidelines on Transparency, 26.

83 Yves Punie et al., DigComp into Action: Get Inspired, Make It Happen. A User Guide to the European Digital Competence Framework (Brussels: Publications Office of the European Union, 2018): 7. 10.2760/112945.

84 See Rossi et al., "When Design Met Law."

85 Reidenberg et al., "Trustworthy Privacy Indicators," 15-6.

86 See the initiatives listed at https:// www.privacyiconsforum.eu/ (accessed November 12, 2019). concepts are more widely known.$^{79}$ In addition, more rigorous assessments of DaPIS must be carried out, including on dimensions such as visibility, ease of learning, culture-independence, and discriminability. ${ }^{80}$ In particular, DaPIS needs to be evaluated according to its function as information markers in a privacy policy. Investigating whether icons can compose the first layer of a layered approach, providing in an "easily visible, intelligible, and clearly legible manner a meaningful overview of the intended processing" and of consent requests, also is necessary. ${ }^{81}$

Further research also should be devoted to the design of information and privacy indicators on small screens, such as tablets and smartphones, but also internet of things (IoT) devices without screens and in surveillance environments.

\section{The Challenge of Universal Interpretation}

For the reasons already explained, expectations that icons can be uniformly and immediately understood by any user must be approached with due precautions. ${ }^{82}$ Nevertheless, widespread recognition can be facilitated by supporting initiatives toward international visual standardization and toward the education of data subjects.

Educational measures could be included in the development of the fundamental digital skills envisioned by the European Digital Framework for Citizens (DigComp). ${ }^{83}$ Already included are skills related to privacy, security, and data protection. The long-term goal is to raise awareness and develop a shared culture on such topics. In the specific context of icon research, such a step arguably would be beneficial to augment familiarity and recognition rates. However, expecting icons to increase people's understanding of data protection issues and to solve the critical transparency problems that privacy-related communication classically poses is simply wrong. In this respect, many other design-based interventions can be developed and experimented with. ${ }^{84}$

International standardization is also a necessary step and has a twofold objective. First, it seeks to limit the proliferation of concurrent icon sets that, after a constructive initial phase of divergent creation, becomes an obstacle to widespread recognition and implementation..$^{85}$ Second, it seeks to increase familiarity with the visual language and the underlying concepts and hence to increase the ease of recognition. Research efforts to create and evaluate a reliable icon system are increasing internationally $y^{86}$; but deciding on one icon set should eventually be the goal, leading to widespread and uniform use, supported by influential actors, such as major companies of the digital economies. Moreover, only the European Commission's adoption of delegated acts can establish the object of representation, the function of icons, and the elements 
of the icon set-possibly with the prior involvement of experts, the consideration of the outcomes of empirically based international studies, and provision of the necessary infrastructure for those international studies.

\section{Conclusions and Future Work}

Can visual design effectively communicate relevant privacy and data protection aspects to members of the public? Can this communication improve data subjects' decision-making about data privacy and the use of their legal rights under the GDPR? This piece provides an overview of the main research challenges posed by the development and evaluation of a data protection icon set, enshrined by the GDPR as a transparency-enhancing mechanism. However, much research lies ahead. The adoption of delegated acts is urged by EU Member States ${ }^{87}$; however, the European Commission should not hurriedly choose one code of icons without appropriate evidence supporting its efficacy for the stated purposes. Instead, the EU Commission should welcome, scrutinize, and even include in its decision-making the outcomes of initiatives that have been supported by a powerful methodology, that present trustworthy and generalizable results, and that involve stakeholders representing various sectors of society, including industrial partners whose endorsement, acceptance, and application of a specific icon set across and beyond the EU borders is crucial. Furthermore, more concerted efforts should be dedicated to the design of a holistic methodology that combines several evaluation indexes (e.g., comprehensibility, learnability, and culture independence).$^{88}$ Without such endeavors, haphazard adoption of one set of icons presents significant risks, including reversal of the GDPR's praiseworthy efforts to enhance transparency and to rebalance digital asymmetries between data subjects and data-gathering organizations.

\section{Acknowledgments}

The research described in this article was supported by the

87 Memo from the General Secretariat of the Council, subj: Preparation of the Council position on the evaluation and review of the General Data Protection Regulation (GDPR)-Comments from Member States 12756/1/19REV 1 (Brussels: Council of the European Union, October 9, 2019): 15, https://data. consilium.europa.eu/doc/document/ ST-12756-2019-REV-1/en/pdf (accessed on 12 November 2019).

88 See Rossi and Lenzini, "Which Properties Has an Icon."
EACEA, financing the Joint International Doctoral Degree in Law, Science and Technology (LAST-JD) and by the Mining and Reasoning with Legal Texts (MIREL) project funded under the Marie Sklodowska-Curie grant agreement No 690974. The authors thank all those who have taken part in this project in various degrees: Margaret Hagan, Legal Design Lab, Stanford University; Professor Danilo Danisi and the students of the Accademia di Belle Arti di Bologna; Chiara Cavazzuti, Accademia di Belle Arti di Firenze; and Miroslav Kurdov, Sketchlex. All the participants in the "Law \& Design for Privacy" workshops and in the user studies, and the anonymous reviewers are gratefully acknowledged. 